\title{
American Option Pricing Using Particle Filtering Under Stochastic Volatility Correlated Jump Model
}

\author{
Bin SONG \\ Investment Department, School of Management Science and Engineering, Central University of \\ Finance and Economics, Beijing 100081, China \\ E-mail: selviasong@163.com \\ Enqi LIANG \\ Derivatives Trading Department, China Securities Co., Ltd., Beijing 100010, China \\ E-mail: enqiliang@163.com \\ Bing LIU \\ Investment Department, School of Management Science and Engineering, Central University of \\ Finance and Economics, Beijing 100081, China \\ E-mail: 834579100@qq.com
}

\begin{abstract}
A particle filter based method to price American option under partial observation framework is introduced. Assuming the underlying price process is driven by unobservable latent factors, the pricing methodology should contain inference on latent factors in addition to the original least-squares Monte Carlo approach of Longstaff and Schwartz. Sequential Monte Carlo is a widely applied technique to provide such inference. Applications on stochastic volatility models has been introduced by Rambharat, who assume that volatility is a latent stochastic process, and capture information about it using particle filter based "summary vectors". This paper investigates this particle filter based pricing methodology, with an extension to a stochastic volatility jump model, stochastic volatility correlated jump model (SVCJ), and auxiliary particle filter (APF) introduced first by Pitt and Shephard. In the APF algorithm of SVCJ model, it also provides a modification version to enhance the performance in the resampling step. A detailed implementation and numerical examples of the algorithm are provided. The algorithm is also applied to empirical data.
\end{abstract}

Keywords American options; sequential Monte Carlo; particle filter; latent variable; stochastic volatility jump; auxiliary particle filter

\section{Introduction}

American options can be exercised at any time from inception to its maturity. American options traded in practice involve in those written on individual equities traded on the American Stock Exchange (AMEX), on index options on the S\&P 100 Index, and on S\&P 500 Index Futures traded on the Chicago Board Options Exchange (CBOE).

Received January 22, 2014, accepted April 29, 2014

Supported by Natural Science Foundation of China (Grant No. 11301560; 71301173); Philosophy and Social Science Planning of Beijing (Grant No. 13JGB018); the MOE Layout Foundation of Humanities and Social Sciences (14YJA790048) 
[4] first introduced the valuation problem of American options by solving an optimal stopping problem of a discounted expectation of the payoff function under a risk-neutral measure. Assuming that all the factors that affect the underlying price process are observable, the optimal stopping problem can be solved using the principles of dynamic programming, semi-analytical approximations as an adjustment from European options, deterministic solution of solving variational inequalities and stochastic solution of Least-Square Monte Carlo approach. "Volatility smile" in most option markets suggests that the volatility is not constant. The inconsistency between observed market data and the constant volatility model stimulates more realistic assumptions to model the underlying process, such as local volatility model class and stochastic volatility model class. Another stylized fact in the market data is the appearance of jumps in prices. Early jump models have been explored by [7], in which the jump part is modeled as a compound Poisson process, and the volatility remains constant. Since then, more general models are proposed, for example, stochastic volatility jump model class, which allows jumps appear in both volatility and price process. [10] described a new radial basis functions (RBFs) algorithm for pricing American options under Merton's jump-diffusion model, which is based on a differential quadrature approach, that allows the implementation of the boundary conditions in an efficient way. The semi-discrete equations obtained after approximation of the spatial derivatives, using RBFs based on differential quadrature are solved, using an exponential time integration scheme. They also illustrate the efficiency and accuracy of this new algorithm.

Partial integro-differential formulations are often used for pricing American options under jump-diffusion models. A survey on such formulations and their numerical methods is presented. A detailed description of six efficient methods based on a linear complementarity formulation and finite difference discretizations is given. Numerical experiments compare the performance of these methods for pricing American put options under finite activity jump models, see [11]. [6] present an upwind difference scheme for the valuation of perpetual American put options, using Heston's stochastic volatility model. The matrix associated with the discrete operator is an M-matrix, which ensure that the scheme is stable. They apply the maximum principle to the discrete linear complementarity problem in two mesh sets and derive the error estimates.

[1] considers the problem of pricing American options when the dynamics of the underlying are driven by both stochastic volatility following a square-root process.

As we have known, the volatility can not be directly observed. Under the framework of partial observation, Sequential Monte Carlo (SMC) is a widely studied algorithm that provides statistical inference on unobservable state-space models. Sequential importance resampling (SIR) algorithm can be used to estimate the latent factors given the observed data. In particular, we discuss how particle filtering, usually considered as the "default choice" of SMC, is implemented. An extension of SIR algorithm, called APF which is introduced by [8]. In the context of American option pricing, [9] is the first to study the particle filter based pricing problem.

\section{American option pricing problem with stochastic volatility jump models}

We employ a hidden Markov model (HMM) to describe the price process driven by unobservable latent factor. Let $(\Omega, \mathcal{F}, \mathbb{P})$ be a probability space satisfying usual conditions. Let 
$\left\{L_{n}\right\}_{n \geq 0}$ and $\left\{S_{n}\right\}_{n \geq 0}$ be vector-valued stochastic processes defined on $(\Omega, \mathcal{F}, \mathbb{P})$. Assume that only $\left\{S_{n}\right\}_{n \geq 0}$, denoted as the dynamic of asset prices, is observable, while the "latent factor" that $\left\{L_{n}\right\}_{n \geq 0}$, which stands for "latent", is unobservable. We denote the lowercase $\left\{s_{n}\right\}_{n \geq 0}$ and $\left\{l_{n}\right\}_{n \geq 0}$ as observed and known data set. Also denote $s_{0: n}: e q q\left\{s_{0}, s_{1}, \cdots, s_{n}\right\}$ and $l_{0: n}: e q q\left\{l_{0}, l_{1}, \cdots, l_{n}\right\}$.

$$
\begin{aligned}
L_{0} & \sim p\left(l_{0}\right) \\
\left.L_{n+1}\right|_{L_{n}=l_{n}} & \sim p\left(l_{n+1} \mid l_{n}\right) \\
\left.S_{n+1}\right|_{L_{1: n+1}=l_{1: n+1}}=\left.S_{n+1}\right|_{L_{n+1}=l_{n+1}} & \sim p\left(s_{n+1} \mid l_{n+1}\right)
\end{aligned}
$$

This class of hidden Markov models (HMM) includes most models of interests in quantitative finance. For example, stochastic volatility models and jump models. Our last goal is to derive an Monte Carlo based algorithm on the valuation of American option.

\subsection{Optimal stopping problem under partial information}

Assume that we have a hidden Markov model $\left\{\left(L_{n}, S_{n}\right), n=0,1, \cdots, T\right\}$ as described in $(1) \sim(3)$ under a finite dimensional time space $\mathcal{T}: e q q\{0,1, \cdots, T\}$. The filtration generated by $\left\{\left(L_{n}, S_{n}\right)\right\}$ is $\mathcal{F}_{n}=\sigma\left\{\left(L_{i}, S_{i}\right) ; i=0,1, \cdots, n\right\}$, and the observable filtration generated by observable data is $\mathcal{F}_{n}^{S}=\sigma\left(S_{0}, S_{1}, \cdots, S_{n}\right)$. A random variable $\tau: \Omega \rightarrow \mathcal{T}$ is a $\mathcal{F}_{n}^{S}$-stopping time if $\{\tau \leq n\} \in \mathcal{F}_{n}^{S}$ for every $n \in \mathcal{T}$. Also define $\mathcal{T}^{S}$ as the set of $\mathcal{F}_{n}^{S}$-stopping times $\tau \in \mathcal{T}$. At time 0 , the initial price $S_{0}$ is known and the latent factor $L_{0}$ follows a known distribution $\pi_{0}=p\left(l_{0}\right)$, which is derived from the historical data up to $S_{0}$.

Let $u_{0}=u_{0}\left(S_{0}, \pi_{0}\right)$ denotes the price of an American option on $S_{0}$. Under certain regular conditions, the fundamental theorem of asset pricing shows that the arbitrage-free price of the option with maturity $T$ is a finite-horizon partially observable optimal stopping problem:

$$
u_{0}\left(S_{0}, \pi_{0}\right)=\sup _{\tau \in \mathcal{T}^{S}} \mathbb{E}^{\mathbb{Q}}\left[\mathrm{e}^{-r \tau} g\left(S_{\tau}, L_{\tau}, \tau\right) \mid S_{0}=s_{0}, L_{0} \sim \pi_{0}\right]
$$

where $g: \mathcal{S} \times \mathcal{L} \times \mathcal{T} \rightarrow \mathbb{R}$ is the payoff function at time $\tau$. For a strike price $K$, the payoff of a call option is $g=g\left(S_{\tau}\right)=\left(S_{\tau}-K\right)^{+}$; and for a put option, $g\left(S_{\tau}\right)=\left(K-S_{\tau}\right)^{+}$. In addition, $\mathbb{E}^{\mathbb{Q}}[\cdot \mid \cdot]$ stands for the conditional expectation under the chosen equivalent martingale measure $\mathbb{Q}$ with respect to $\mathbb{P}$. In this setting, the decision maker has only access to $S_{n}$ at time $n$, so that the decision is made only relying on $\mathcal{F}_{n}^{S}$.

According to [12], the above partially observable problem can be transformed to an equivalent fully observable form by introducing a new state "filtering distribution", denoted as $\Pi_{n}=p\left(L_{n} \mid s_{0: n}\right)$, which can be estimated by sequential Monte Carlo techniques. Given $\left(S_{n}, \Pi_{n}\right)$, the optimal pricing problem is equivalent to

$$
u_{0}\left(S_{0}, \pi_{0}\right)=\sup _{\tau \in \mathcal{T}^{S}} \mathbb{E}^{\mathbb{Q}}\left[\mathrm{e}^{-r \tau} \tilde{g}\left(S_{\tau}, \Pi_{\tau}, \tau\right) \mid S_{0}=s_{0}, L_{0} \sim \pi_{0}\right]
$$

where

$$
\tilde{g}\left(S_{n}, \Pi_{n}, n\right)=\mathbb{E}\left[g\left(S_{n}, L_{n}, n\right) \mid \mathcal{F}_{n}^{S}\right]=\int g\left(L_{n}, S_{n}\right) \Pi_{n}\left(L_{n}\right) \mathrm{d} L_{n}
$$

Theoretically, we can solve it following the dynamic programming recursion:

$$
u_{n}\left(S_{n}, \Pi_{n}\right)=\max \left(\tilde{g}\left(S_{n}, \Pi_{n}, n\right), C_{n}\left(S_{n}, \Pi_{n}, n\right)\right), n=T, \cdots, 1
$$


where $C_{n}\left(S_{n}, \Pi_{n}, n\right)$ is the continuation value at time $t$ defined as

$$
\begin{aligned}
C_{T}\left(S_{T}, \Pi_{T}, T\right) & =\tilde{g}\left(S_{T}, \Pi_{T}, T\right) \\
C_{n}\left(S_{n}, \Pi_{n}, n\right) & =\mathbb{E}\left[u_{n+1}\left(S_{n+1}, L_{n+1}, n+1\right) \mid S_{n}, \Pi_{n}\right], n=T-1, \cdots, 0
\end{aligned}
$$

Then the optimal stopping time is

$$
\tau^{*}=\min \left\{t \in \mathcal{T} \mid \tilde{g}\left(S_{n}, \Pi_{n}, n\right)>C_{n}\left(S_{n}, \Pi_{n}, n\right)\right\}
$$

The above recursion show that $\left(S_{n}, \Pi_{n}\right)$ are sufficient statistics that determine the optimal stopping time. However, it is often impossible to solve the problem exactly following (7). The difficulty inside comes first from the fact that the filtering distribution $\Pi_{n}$ is infinite dimensional, and that a lack of accurate estimation of continuation value $C_{n}\left(S_{n}, \Pi_{n}, n\right)$. To overcome these two problems, Monte Carlo technique can be used to provide approximation. This is essentially done by firstly using particle filter to approximate the filtering distribution $\Pi_{n}$, and summarize it within a finite-dimensional vector that describes the statistical property of it. Then a

least-square Monte Carlo can be applied to estimate $C_{n}\left(S_{n}, \Pi_{n}, n\right)$. The detail algorithm is introduced in the next section.

\subsection{Generic particle filter based American options pricer}

We combine the dynamic programming algorithm with sequential Monte-Carlo techniques to pricing American options by particle filtering. The key to solve (7) is to provide a finite dimensional summary vector $\pi_{n}^{m}$ of from $m$ filtered particles at time $n$. The filtering distribution vary from time to time. For stochastic volatility model, the posterior distribution is usually near to the previous one. In such a case, measures of location and scale would be sufficient. The posterior distribution of volatility of the jump models can be highly skewed and may contains multiple peaks, especially when a jump in volatility and price occurs. In such a case, one may think of taking two modes of the filtered particles.

Assumes that under our algorithm, we have $M$ scenarios indexed by $i$, with $N$ time steps indexed by $j$, and $m$ particles in each step indexed by $k$. For sake of simplicity, we present the SIR version here. Since the filtering step can be separated from the backward decision step, it is straightforward to implement the corresponding APF version.

Assume that under the physical measure $\mathbb{P}$, the asset price $S_{t}$ is driven by two latent factors, namely, the latent variance and the latent jump process. Specifically, we assume that they are the solutions of the following stochastic differential equations:

$$
\begin{aligned}
& \frac{\mathrm{d} S_{t}}{S_{t-}}=\mu \mathrm{d} t+\sqrt{V_{t-}} \mathrm{d} W_{t}^{s}+\left(\mathrm{e}^{Z_{t}^{s}}-1\right) \mathrm{d} N_{t} \\
& \mathrm{~d} V_{t}=\kappa\left(\theta-V_{t-}\right) \mathrm{d} t+\eta \sqrt{V_{t-}} \mathrm{d} W_{t}^{v}+Z_{t}^{v} \mathrm{~d} N_{t} \\
& \mathbb{E}\left[\mathrm{d} W_{t}^{s} \mathrm{~d} W_{t}^{v}\right]=\rho \mathrm{d} t
\end{aligned}
$$

Where $W=\left(W_{t}^{s}\right)_{t \geq 0}$ and $W=\left(W_{t}^{v}\right)_{t \geq 0}$ are two Brownian motions under probability measure $\mathbb{P}$, with parameters $\kappa, \theta, \eta$ and $\rho$ are the same as Heston model. In addition, we assume that the jump sizes are $Z_{t}^{s} \sim \mathcal{N}\left(\mu_{s}, \sigma_{s}^{2}\right), Z_{t}^{v} \sim \exp \left(\mu_{v}\right)$ with correlation $\rho_{z}$. The jump times $N_{t} \sim \operatorname{Poi}\left(\lambda_{t}\right)$ is a Poisson process with intensity $\lambda_{t}$ for both spot and variance. This implicates the spot and variance would jump simultaneously, which is coherent with listed option data. For 
sake of simplicity, we assume the independence of jump and spot volatility, i.e. the correlation $\rho_{z}=0$, because this parameter is difficult to estimate, as argued by [3] and [2].

\subsection{Particle filtering in stochastic volatility jump model}

The only difference between SVCJ and SV model is to simultaneously sample two latent factors and calculate the importance density in a more careful way. In SIR algorithm, the derivation is more straghtforward. The APF algorithm is more challenging because by first resampling, posterior distributions become some normal mixture.

\subsubsection{Discretization of SVCJ model}

Denote $y_{n+1}=\ln \left(S_{n+1} / S_{n}\right)$ as the log-return of asset price. By Euler-Maruyama scheme with sufficiently small equidistant time step $h$, we can discretize (11) (13) associated with the discretely observed data by

$$
\begin{aligned}
y_{n+1}= & \sum_{j=1}^{n_{h}}\left(\mu-\frac{V_{n+j h}}{2}\right) h+\sum_{j=1}^{n_{h}} \sqrt{V_{n+j h}}\left(\sqrt{1-\rho^{2}} \Delta W_{n+j h}^{(1)}+\rho \Delta W_{n+j h}^{(2)}\right) \\
& +\sum_{j=1}^{n_{h}} Z_{n+j h}^{s} J_{n+j h} \\
V_{n+1}= & V_{n}+\sum_{j=1}^{n_{h}} \kappa\left(\theta-V_{n+j h}\right) h+\sum_{j=1}^{n_{h}} \eta \sqrt{\left|V_{n+j h}\right|} \Delta W_{n+j h}^{(2)}+\sum_{j=1}^{n_{h}} Z_{n+j h}^{v} J_{n+j h}
\end{aligned}
$$

where $\left(\Delta W_{n+j h}^{(1)}, \Delta W_{n+j h}^{(2)}\right)$ are two independent Brownian motions, and $J_{n+j h} \sim \operatorname{Ber}\left(\lambda_{t}\right)$ are Bernoulli random variables with intensities $\lambda_{t}$. The jump size is consistent over all timediscretization choice. We summarize the latent factors by

$$
L_{n}=\left(V_{n}, Z_{n}^{s}, Z_{n}^{v}, J_{n}\right)
$$

It is easy to show that $\pi_{0}=p\left(l_{0}\right)=p\left(V_{0}\right) \sim \chi^{2}\left(\frac{2 \kappa \theta}{\eta^{2}}, \frac{\eta^{2}}{2 \kappa}\right)$. We still need to specify $\Pi_{n}=$ $p\left(L_{n} \mid s_{0: n}\right)=p\left(L_{n} \mid y_{0: n}\right)$ by sequential Monte Carlo.

\subsubsection{APF algorithm for SVCJ model}

For APF algorithm, we need to specify how to compute the importance weights via $p\left(y_{n+1} \mid L_{n}\right)$, and sample from $p\left(L_{n+1} \mid L_{n}, y_{n+1}\right)$ to obtain an empirical approximation of $\Pi_{n+1}$. The first step to evaluate $p\left(y_{n+1} \mid L_{n}\right)$ can be separated via

$$
p\left(y_{n+1} \mid L_{n}\right)=\int p\left(y_{n+1} \mid L_{n: n+1}\right) p\left(L_{n+1} \mid L_{n}\right) \mathrm{d} L_{n: n+1}
$$

Notice that the conditional distributions of $p\left(y_{n+1} \mid L_{n+1}\right)$ are Gaussian. The approximation of $p\left(y_{n+1} \mid L_{n: n+1}\right)$ is straight forward from

$$
p\left(y_{n+1} \mid L_{n: n+1}^{(k)}\right) \simeq \phi\left(y_{n+1} \mid \mu_{y}^{(k)},\left(\sigma_{y}^{(k)}\right)^{2}\right)
$$

To approximate $p\left(L_{n+1} \mid L_{n}\right)$, we need to be more careful to deal with jumps of spot variance, because the jumps of asset prices do not have an influence on it. Following the mind as SV 
model, we need to compute the predictive particles $\hat{V}_{n+j h}^{(k)}$. One way to do so is to compute the conditional expectation of $\mathbb{E}\left[V_{n+j h} \mid V_{n}\right]$ by

$$
\hat{V}_{n+j h}^{(k)}=\mathbb{E}\left[V_{n+j h} \mid V_{n}^{(k)}\right]=V_{n}^{(k)}+\kappa\left(\theta-V_{n}^{(k)}\right) j h+\lambda \mu_{v}
$$

This is the most referred approach in current literature. However, it is essentially just a movement for all particles, and may suffer from sample impoverishment problem. We propose another way to calculate this predictive density. We thus have the approximation of (17) with

$$
\begin{aligned}
& p\left(y_{n+1} \mid L_{n}^{(k)}\right) \\
\simeq & \sum_{i=0}^{\infty} \phi\left(y_{n+1} \mid \sum_{j=0}^{n_{h}}\left(\mu-\frac{\hat{V}_{n+j h}^{(k)}}{2}\right) h+i \mu_{s}, \sum_{j=0}^{n_{h}} \hat{V}_{n+j h}^{(k)}\left(1-\rho^{2}\right) h+i \sigma_{s}^{2}\right) \frac{\lambda^{i} \mathrm{e}^{-\lambda}}{i !} \\
\simeq & \lambda \phi\left(y_{n+1} \mid \sum_{j=0}^{n_{h}}\left(\mu-\frac{\hat{V}_{n+j h}^{(k)}}{2}\right) h+\mu_{s}, \sum_{j=0}^{n_{h}} \hat{V}_{n+j h}^{(k)}\left(1-\rho^{2}\right) h+\sigma_{s}^{2}\right) \\
& +(1-\lambda) \phi\left(y_{n+1} \mid \sum_{j=0}^{n_{h}}\left(\mu-\frac{\hat{V}_{n+j h}^{(k)}}{2}\right) h, \sum_{j=0}^{n_{h}} \hat{V}_{n+j h}^{(k)}\left(1-\rho^{2}\right) h\right)
\end{aligned}
$$

where $\phi\left(\cdot \mid \mu, \sigma^{2}\right)$ is a normal density. Then we can resample particles $L_{n}^{(k)}$ using $p\left(y_{n+1} \mid L_{n}^{(k)}\right)$.

The second step is to update the particles from $p\left(L_{n+1} \mid L_{n}, y_{n+1}\right)$. In SVCJ model, this remains to sample three variables: the jump times, the jump sizes, and the spot variance. Since the latter two depend on the first, we can simulate the jump times marginalizing out jump sizes:

$$
\begin{aligned}
& p\left(J_{n+1}^{(k)}=i \mid V_{n}^{(k)}, y_{n+1}\right) \\
\propto & p\left(y_{n+1} \mid J_{n+1}^{(k)}=i,\left\{\hat{V}_{n+j h}^{(k)}\right\}\right) p\left(J_{n+1}^{(k)}=i\right) \\
\propto & \phi\left(y_{n+1} \mid \sum_{j=0}^{n_{h}}\left(\mu-\frac{\hat{V}_{n+j h}^{(k)}}{2}\right) h+i \mu_{s}, \sum_{j=0}^{n_{h}} \hat{V}_{n+j h}^{(k)}\left(1-\rho^{2}\right) h+i \sigma_{s}^{2}\right) \frac{\lambda^{i} \mathrm{e}^{-\lambda}}{i !} \\
\simeq & \left\{\begin{array}{l}
\lambda \phi\left(y_{n+1} \mid \sum_{j=0}^{n_{h}}\left(\mu-\frac{\hat{V}_{n+j h}^{(k)}}{2}\right) h+\mu_{s}, \sum_{j=0}^{n_{h}} \hat{V}_{n+j h}^{(k)}\left(1-\rho^{2}\right) h+\sigma_{s}^{2}\right), i=1 \\
(1-\lambda) \phi\left(y_{n+1} \mid \sum_{j=0}^{n_{h}}\left(\mu-\frac{\hat{V}_{n+j h}^{(k)}}{2}\right) h, \sum_{j=0}^{n_{h}} \hat{V}_{n+j h}^{(k)}\left(1-\rho^{2}\right) h\right), \quad i=0
\end{array}\right.
\end{aligned}
$$

The last step is a Bernoulli approximation of Poisson distribution. For sufficiently small $\lambda \leq 0.1$, which is usually the case in empirical study, these two do not distinguish themselves. With the approximation of jump times $J_{n+1}^{(k)}$, we can then sample jump sizes for those particles with $J_{n+1}^{(k)} \geq 1$. Thanks to the normal assumption of jump size, this can be easily done via a well-known property that conditional normal distribution is again of a form of normal distribution. Given the jump times $J_{n+1}^{(k)}$, we have $Z_{n+1}^{s} \sim \mathcal{N}\left(J_{n+1}^{(k)} \mu_{s}, J_{n+1}^{(k)} \sigma_{s}^{2}\right)$, and $y_{n+1} \sim$ $\mathcal{N}\left(\sum_{j=0}^{n_{h}}\left(\mu-\frac{\hat{V}_{n+j h}^{(k)}}{2}\right) h+J_{n+1}^{(k)} \mu_{s}, \sum_{j=0}^{n_{h}} \hat{V}_{n+j h}^{(k)}\left(1-\rho^{2}\right) h\right)$, and thus can represent the conditional 
distribution by another normal distribution:

$$
p\left(Z_{n+1}^{s} \mid J_{n+1}^{(k)}, y_{n+1}\right)=p\left(y_{n+1} \mid J_{n+1}^{(k)}, Z_{n+1}^{s}\right) \frac{p\left(Z_{n+1}^{s}, J_{n+1}^{(k)}\right)}{p\left(y_{n+1}, J_{n+1}^{(k)}\right)}=\phi\left(\mu_{z}^{(k)},\left(\sigma_{z}^{(k)}\right)^{2}\right)
$$

where the mean and variance is given by

$$
\begin{aligned}
\mu_{z}^{(k)} & =J_{n+1}^{(k)} \mu_{s}+\frac{J_{n+1}^{(k)} \sigma_{s}^{2}}{\sum_{j=0}^{n_{h}} \hat{V}_{n+j h}^{(k)}\left(1-\rho^{2}\right) h}\left(y_{n+1}-\sum_{j=0}^{n_{h}}\left(\mu-\frac{\hat{V}_{n+j h}^{(k)}}{2}\right) h-J_{n+1}^{(k)} \mu_{s}\right) \\
\left(\sigma_{z}^{(k)}\right)^{2} & =J_{n+1}^{(k)} \sigma_{s}^{2}\left(1-\frac{J_{n+1}^{(k)} \sigma_{s}^{2}}{\sum_{j=0}^{n_{h}} \hat{V}_{n+j h}^{(k)}\left(1-\rho^{2}\right) h}\right)
\end{aligned}
$$

For the spot variance, we have a similar formula with stochastic volatility:

$$
p\left(V_{n+1} \mid L_{n}, y_{n+1}\right) \propto p\left(V_{n+1} \mid L_{n}\right) p\left(y_{n+1} \mid V_{n+1}\right)
$$

\subsubsection{Modification of APF in SVCJ model}

APF algorithm can overcome sampling impoverishment problem by first resampling. However, in the resampling step of spot variance in APF, the conditional expectation as the predictive sample, which may again suffers from such a problem. The problem arises in SVCJ model when the jumps in volatility occur. When there is a jump in volatility, the distribution of post-jump volatility changes dramatically comparing to the pre-jump volatility, and there is a probability that a finite-dimensional sample space does not contain the post-jump particles. We consider the following example.

Consider the case that at time $n$, we have a spot volatility $V_{n}=4$, and a jump occurs with $Z_{n+1}^{v}=10$, resulting in a post-jump volatility to be $V_{n+1}=14$ and observed return $y_{n+1}=-15$. A typical view of such a case would looks like the first panel in Figure 1.
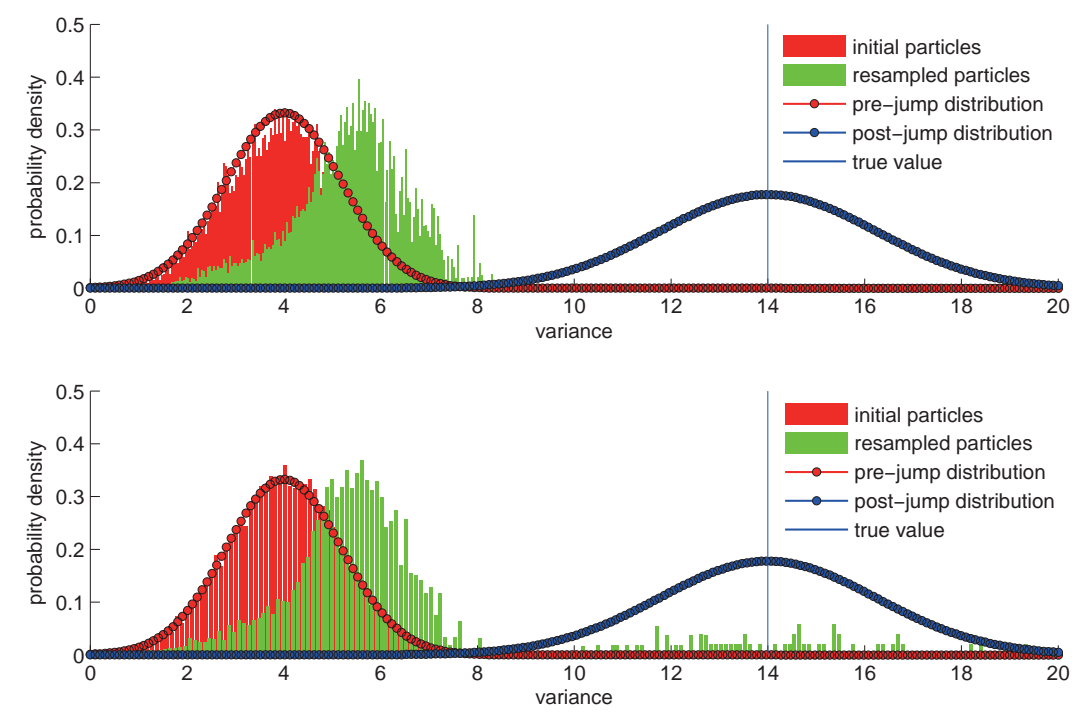

Figure 1 Illustration of predictive particles calculated via conditional expectation in APF 
In Figure 1, we have some initial particles $\left\{V_{n}^{(k)}\right\}$ as histogramed in red. The corresponding probability distribution of these particles is non-central $\chi^{2}$-distribution, which looks similar with normal distribution, as plotted in red dash-dot line. Now a jump occured at a size of $Z_{n+1}^{v}=10$, leads to the updated true volatility value to be $V_{n+1}=14$, plotted in a blue line. The corresponding probability density function conditioned on the jump occured is plotted in blue dash-dot line. By Bayesian formula, we can update the initial particles incorporating the new information $y_{n+1}=-15$, using first the conditional expectation (19) to "esimate" the volatilities, then calculate the importance weights using (20), and resampling according to the weights. The posterior resampled particles $\left\{V_{n+1}^{i(k)}\right\}_{k=1}^{m}$ becomes then the reweighted version of initial particles.

However, none of the resampled particles $\left\{V_{n+1}^{i(k)}\right\}_{k=1}^{m}$ contains, or even be near to, the true post-jump value of volatility. Although we give a large weight for the maximum value of initial particle, and obtain some particles with a value around 8.25, we cannot capture any postjump particle. The interpretation is quite straghtforward: the prediction step using conditional expectation (19) just shifts forward the particle a bit, and the resampling step is just to reweight the probability of the existed particles and thus does not change the sample state space of the particles. As a result, if a jump does occur, neither do the two steps would create reasonable particles for the post-jump volatility.

To overcome this problem, we can think of a scheme to diversify the particles by adding some particles conditioning on the case when we have jumps. Assume that we have a probability of jump $\lambda$, we can assign some of the particles to be having some jumps. Precisely, we can assign a number $k_{\lambda}$ with the form

$$
k_{\lambda}=\max \{1,[\lambda \cdot m]\}
$$

where $[x]$ stands for taking the floor of $x$ to make it an integer, and compute the predictive particles by

$$
\hat{V}_{n+1}^{(k)}=V_{n}^{(k)}+\kappa\left(\theta-V_{n}^{(k)}\right)+Z_{n+1}^{v,(k)}
$$

for $k=1, \cdots, k_{\lambda}$ randomly chosen, and the jumps $Z_{n+1}^{v,(k)} \sim \exp \left(\mu_{v}\right)$. The intuition behind (24) is quite straightforward: we add at least one particle with jump, and add more if we have enough total particles. With this, we can diversify the particles by manually assigning some particles with jumps. In our example here, the result can be shown in panel 2 of figure 1 .

In the panel 2 of figure 1 , we first sample $\hat{V}_{n+1}^{(k)}$ from (25), and compute the importance weights as in (20) and resample. Not surprisingly, we add some diversification on particles, in the sense that they contains the region of "true post-jump volatility". Notice that the resampled particles do not typically concentrate on some single point, even at the point where the true value stays. This is a well-known effect shown by many Bayesian statistical problems, that the historical data would tend to reject the posterior distribution and make a compromise in between. In the example here, since the probability of jumps is quite low, the model made a compromise in such a way that it increases almost all the numbers of with-jump particles (comparing to one particle in before), but with a highly non-normal posterior density.

This would introduce new challenges in the American option pricing problem, in which we have to summarize particles in each step. 


\subsubsection{Numerical experiment}

We now show some numerical results on the stochastic volatility model with jumps. The parameters are as follows:

Table 1 Parameters of SVCJ model in the numerical example

\begin{tabular}{ccccccccc}
$\kappa$ & $\theta$ & $\eta$ & $\rho$ & $\lambda$ & $\mu_{S}$ & $\sigma_{S}$ & $\mu_{V}$ & $T$ \\
\hline 0.02 & 0.9 & 0.15 & 0 & 0.006 & -2.5 & 4 & 2 & 2000 \\
\hline
\end{tabular}

The parameters in Table 1 are based on [3], and a larger observation period $T=2000$ can capture more jumps in the simulation. We show an comparison of performances for all models by RMSE and MAE. For each model, we implemented the SIR algorithm, the original APF algorithm (APFo) as proposed by [13] and [5], and the modification of APF algorithm (APFm). The main results are shown in Table 2 .

Table 2 Simulation results for SVCJ model

\begin{tabular}{|c|c|c|c|c|c|c|}
\hline \multirow{2}{*}{$m$} & \multicolumn{2}{|c|}{ SIR } & \multicolumn{2}{|c|}{ APFo } & \multicolumn{2}{|c|}{ APFm } \\
\hline & $\mathrm{R}^{1}$ & M & $\mathrm{R}$ & M & $\mathrm{R}$ & M \\
\hline \multicolumn{7}{|c|}{ Variances $V_{n}$} \\
\hline 100 & 12.45 & 8.15 & 13.01 & 8.39 & 11.52 & 7.41 \\
\hline 1000 & 13.15 & 6.93 & 10.90 & 6.85 & 9.77 & 6.26 \\
\hline 10000 & 11.12 & 6.70 & 10.96 & 6.90 & 9.58 & 6.29 \\
\hline \multicolumn{7}{|c|}{ Jumps in price $J_{n} \cdot Z_{n}^{s}$} \\
\hline 100 & 3.54 & 0.27 & 2.34 & 0.26 & 2.74 & 0.29 \\
\hline 1000 & 3.40 & 0.28 & 2.16 & 0.25 & 2.12 & 0.23 \\
\hline 10000 & 3.19 & 0.28 & 2.05 & 0.22 & 2.06 & 0.22 \\
\hline \multicolumn{7}{|c|}{ Jumps in volatility $J_{n} \cdot Z_{n}^{v}$} \\
\hline 100 & 2.44 & 0.23 & 2.29 & 0.25 & 2.00 & 0.21 \\
\hline 1000 & 3.11 & 0.28 & 1.94 & 0.22 & 1.94 & 0.22 \\
\hline 10000 & 1.82 & 0.20 & 1.91 & 0.21 & 1.88 & 0.21 \\
\hline
\end{tabular}

\footnotetext{
${ }^{1} \mathrm{R}$ and $\mathrm{M}$ are RMSE and MAE errors between filtered mean and the true simulation values. Parameters are taken from Table 2 with $n_{h}=10$. The numbers are multiplied by 10 .
}

In Table 2, in general, APFm outperforms the other two. Comparing the RMSE of filter variance, for example, we can observe that when a particle number of 10000 is used, which we think can eliminate the Monte Carlo approximation error, the RMSE is near to 1. However, we can find that the APF algorithm is not always performing better than SIR, especially when the jump intensity is large, say 0.2. This is because we are using an approximation of Bernoulli distribution rather than Binomial to compute the importance weights of jumps, and thus would fail when the jump occurs twice. But for daily return, the event is so rare that it is almost negligible. 
In Figure 2, panel 1 4 show the daily return, spot volatility, jumps in price and jumps in volatility respectively. Both SIR and the modified APF algorithm are implemented. The price process is simulated from SVCJ model, which makes it more difficult to "disentangle" the jumps from the volatility comparing to SVJ model.
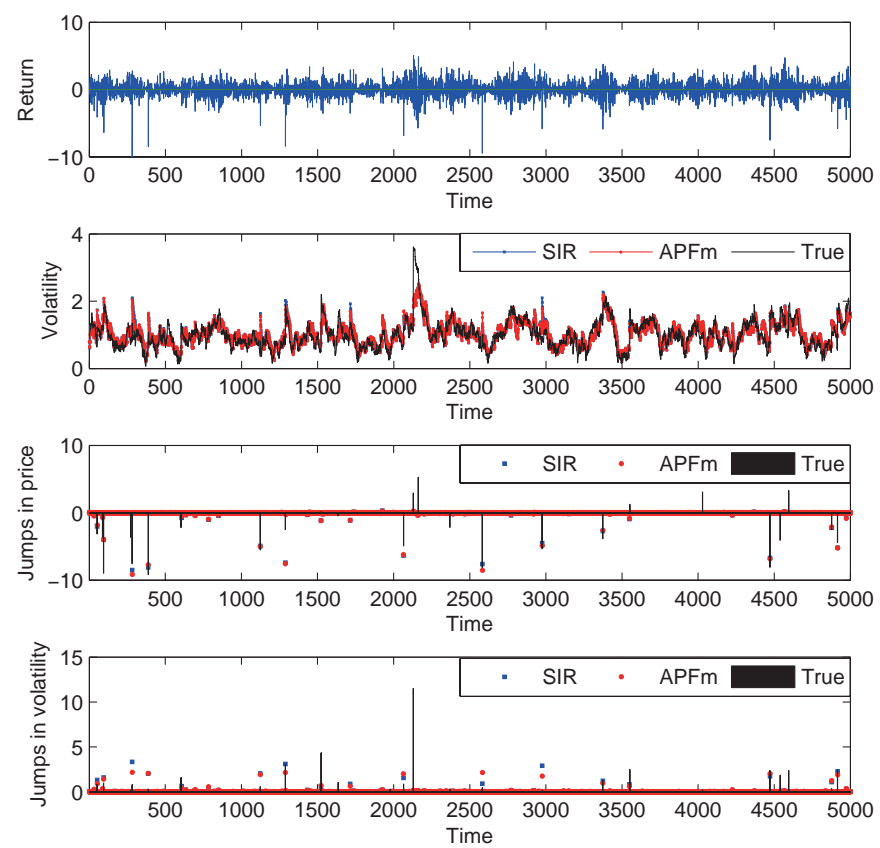

Figure 2 A representative sample path of daily returns, volatility, jumps in prices and jumps in volatility with corresponding filtered estimators. Parameters are taken from

Table 2 with $m=10000, n_{h}=100$ and $T=5000$.
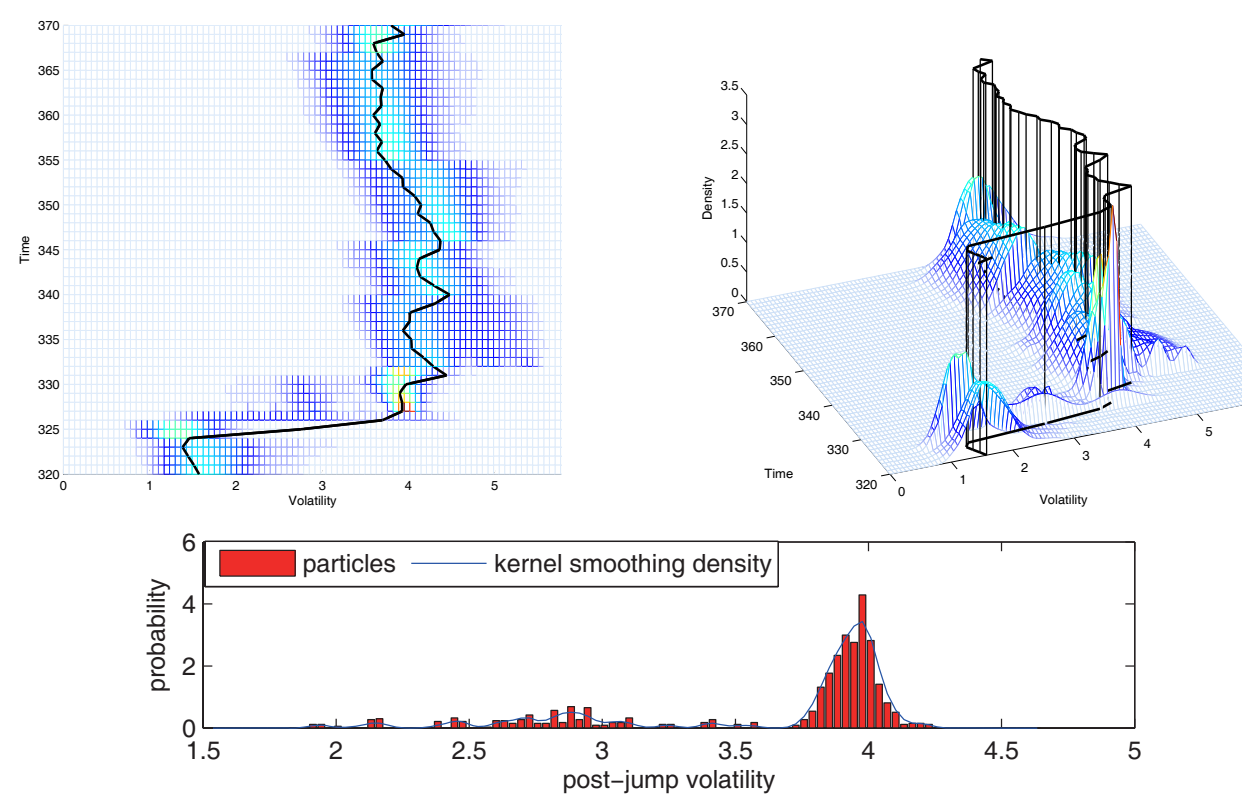

Figure 3 Illustrative filtering distributions over time 
Generally, both SIR and the APFm algorithm follow the true volatility trend. The APFm outperforms SIR in general. It would provide some possibly unreasonable jump particles and thus predict small jumps in volatility. Figure 3 shows the evolution of particles over time. The algorithm is run with APFm with $m=10000$ particles. At time $n=325$, a jump in both price and volatility occurs, and the posterior distribution shifts dramatically. The histogram provides the posterior density at this time. Initially, the particles are centered around 2.8, and the distribution looks like that from stochastic volatility model. When a jump occurred and is detected, the importance weights favors towards the new post-jump value, with a resistency from history. In such a scenario, none of any traditional statistical measure provides an accurate estimation.

\section{Empirical results}

We apply the particle filter based American option pricing algorithm to market data, including SV, SVJ and SVCJ models. We first apply particle filter method to estimate the volatility of S\&P 500 return, and calibrate the risk premium by American style index future options, then compare the historical volatility with the future volatility introduced by VIX; then we compute the cross sectional option prices for different maturities and moneyness to find an inconsistency among options with different maturities. The calibration include two parts: first, we use particle filter to calibrate our model parameter under historical measure using observed underlying market data; second, we calibrate the risk premium associated with the latent factors through minimizing the MSE of model prices and market prices. We also examine the out-of-sample performance of our model.

\subsection{Applications with S\&P 500 returns}

we utilize pure particle filter over SV, SVJ and SVCJ models under historical measure $\mathbb{P}$. The data set we consider are from 01/02/1986 to 01/02/2013. We use data from 01/02/1986 to $12 / 31 / 2004$ of S\&P 500 returns to calibrate the parameter, and implement particle filter with SV, SVJ and SVCJ models for the period during 01/02/1990 to 12/31/2012 since VIX index are available only from $01 / 02 / 1990$. We calibrate the model using SA algorithm for SV, SVJ and SVCJ model, the estimated parameters are given as follows:

Table 3 Estimated parameters for SV, SVJ and SVCJ model

\begin{tabular}{cccccc}
\hline & $\kappa$ & $\theta$ & $\eta$ & $\rho$ & $\mu$ \\
\hline SV & 0.0607 & 1.8082 & 0.3526 & -0.2038 & 0.0618 \\
SVJ & 0.0170 & 0.9121 & 0.1675 & -0.2885 & 0.0861 \\
SVCJ & 0.0180 & 1.2381 & 0.1216 & -0.4082 & 0.0299 \\
\hline & $\lambda$ & $\mu_{S}$ & $\sigma_{S}$ & $\mu_{V}$ & \\
\hline SVJ & 0.0179 & -1.5050 & 4.4196 & & \\
SVCJ & 0.0075 & -4.2116 & 4.3904 & 3.8402 & \\
\hline
\end{tabular}

Parameters are calibrated using S\&P 500 daily returns during the period $01 / 02 / 1986$ to $12 / 31 / 2004$. Simulated annealing algorithm is applied to maximize the likelihood function. 
Then we utilize particle filtering to the subsequent observed return, and the result is shown as in Figure 4.
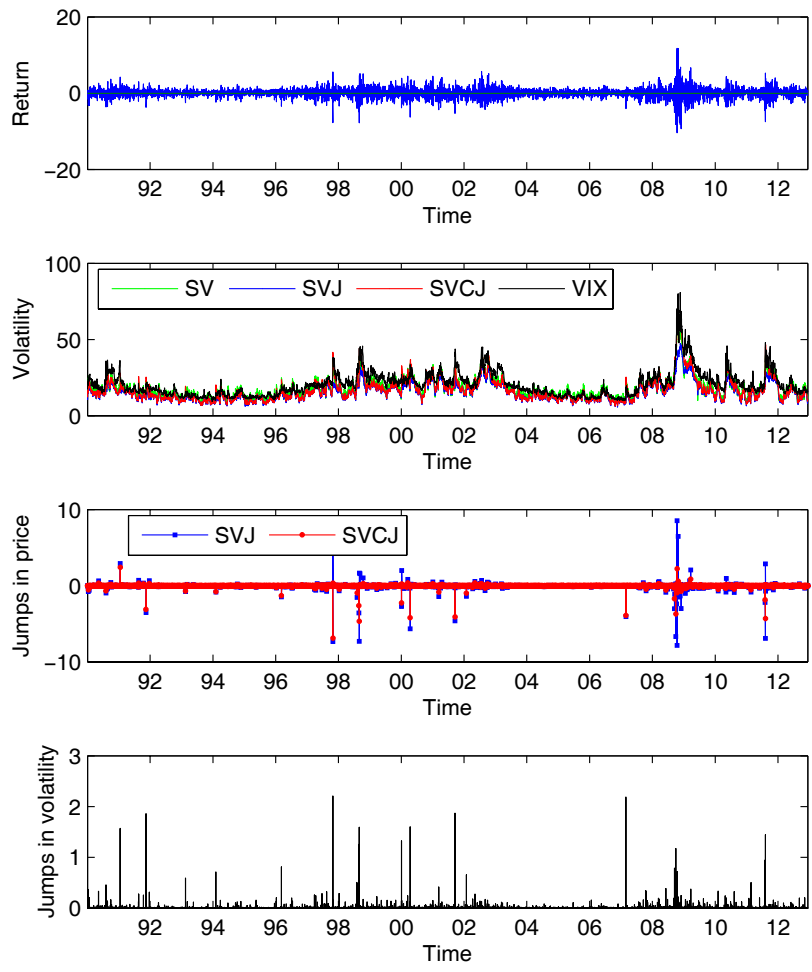

Figure 4 Estimated latent factors for SV, SVJ and SVCJ model for S\&P 500 return, a modified APF algorithm with $m=10000$ particles is used.

Comparing the spot volatility, SV model provides a biggest filtered value, because the other two will "smooth" it by introducing jumps. For SVJ and SVCJ model, we observe that the volatility in SVCJ model would generally be larger than the SVJ model. This can be interpreted by the fact that the jumps in volatility would generally make the contribution of volatility to the price change larger. Numerically, since the jump of volatility can only be positive, the volatility in SVCJ should also be larger.

For the option pricing problem, we consider the stochastic volatility model for S\&P 500 index future returns and index future options, because the algorithm is quite slow and is impractical to estimate any parameters from a large data set. The S\&P 500 index future options are American style.

we take the most active S\&P 500 future contract defined by Bloomberg with ticker being "SP1 Index", the time period is from the first trading day of 2005 to the first trading day of 2013 in total 2,000 observations. The data are obtained from Bloomberg ${ }^{\circledR}$. For the options data, we utilize a scheme to fetch the data. Precisely, we search for those options that are nearest to be at-the-money, and with the nearest time-to-maturity being 30 days. These options are typically most actively traded. For the rates, we use one-month USD Libor rate as the risk-free rate to match the maturities, and Bloomberg best expectation of dividends to approximate the 
dividend yields.

We calibrate the constant volatility risk premium introduced by option markets. we fix all the parameters for the stochastic volatility model, and calibrate the volatility risk-premium through a constant $\lambda^{\mathbb{Q}}$, by minimizing the normalized Mean Squared Error (MSE) of the model price and market observed price:

$$
M S E: \frac{1}{N} \sum_{t \in \mathcal{T}}\left(P_{t}^{M o d}-P_{t}^{M k t}\right)^{2}
$$

This method takes a long time to be done, as we have 2000 observations, and to price a single American option using limited samples and particles, e.g. $M=100, N=100, m=100$, takes already 10 seconds in Matlab. After calibration, we obtain a volatility risk premium being $\lambda=-4.2496 \%$. The result is shown in Figure 5 .
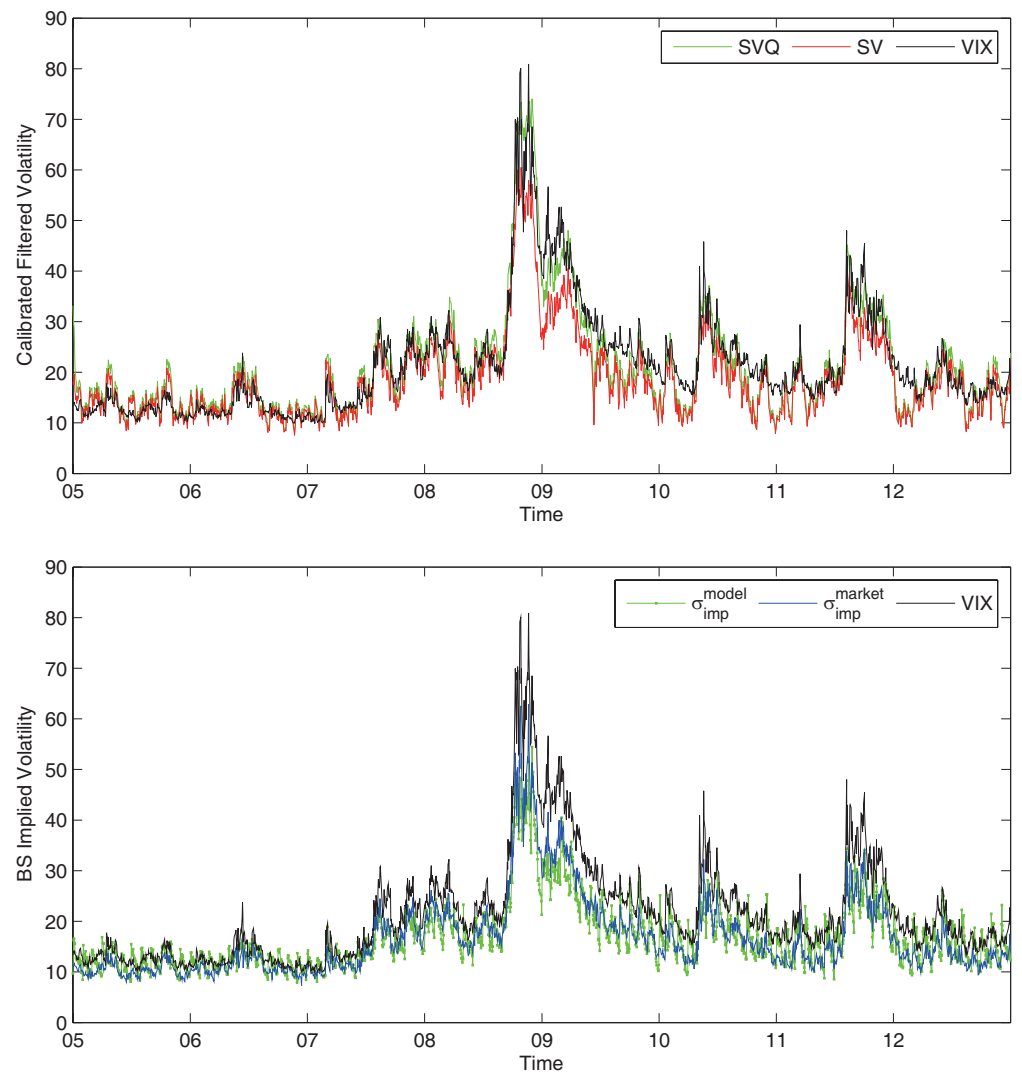

Figure 5 Calibrated risk-neutral filtered volatility v.s. VIX index

In panel 1 of Figure 5, we plotted: 1 . the calibrated filtered volatility with volatility premium of $\lambda=-4.2496 \%$, denoted by SVQ; 2 . the pure particle filtered volatility, denoted by SV; 3 . the VIX index. After calibration, SVQ is generally closer to VIX index, especially during the financial crisis, though bias still exists especially because we have only one parameter changed. The RMSE of SVQ is 20.7853 compared to 21.6994 of SV.

In panel 2 of Figure 5, we plotted: 1. the Black-Scholes implied volatility of predicted price, computed via calibrated volatility using our algorithm; 2. the Black-Scholes implied volatility 
of the market prices, which is different from deriving directly from an European option; 3 . the VIX index. We also plot the VIX index. To the S\&P 500 index future options, it seems that the volatility risk premium has changed over period, because we observe an inconsistency through the time, especially before and after the global financial crisis at 2008, of which the sign being Lehman's bankruptcy on September 15, 2008. In order to study further the volatility risk premium, we adopt another set of most liquidly traded American options written on Apple Inc.

\subsection{Application to American options written on equities}

In this section, we apply the particle filter to some other more liquidly traded options. We use daily closing price of Apple Inc. that we obtain also from Bloomberg ${ }^{\circledR}$. Apple Inc. is one of the largest companies in the world and its options are among in the most liquid. The data we used for estimating the stochastic volatility under historical measure is from $01 / 03 / 2012$ to $10 / 30 / 2012$. We choose this period because it goes through a relatively complete cycle.

We also obtain the American put option prices from Bloomberg ${ }^{\circledR}$. During that period, the most actively traded options are $\mathcal{K}=\{550,555,560,565,570\}$ with maturities $\mathcal{T}=\{12 / 22 / 2012$, $01 / 19 / 2013,02 / 16 / 2013\}$, so we have 15 options at each day. The filtered volatility of Apple Inc. is given in Figure 6 .
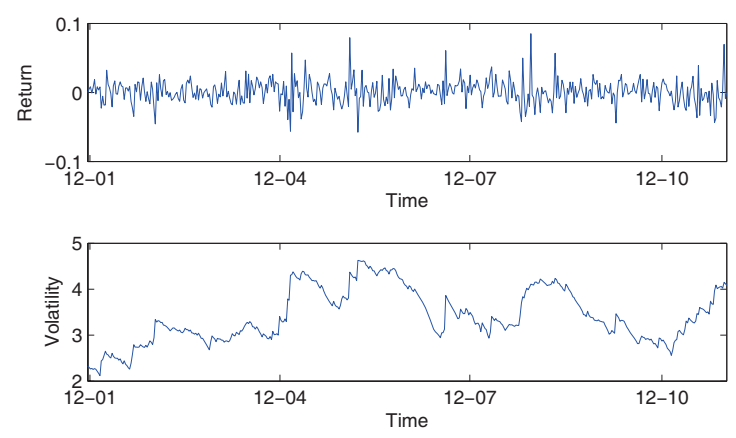

Figure 6 Apple daily closing prices and filtered volatility under stochastic volatility model

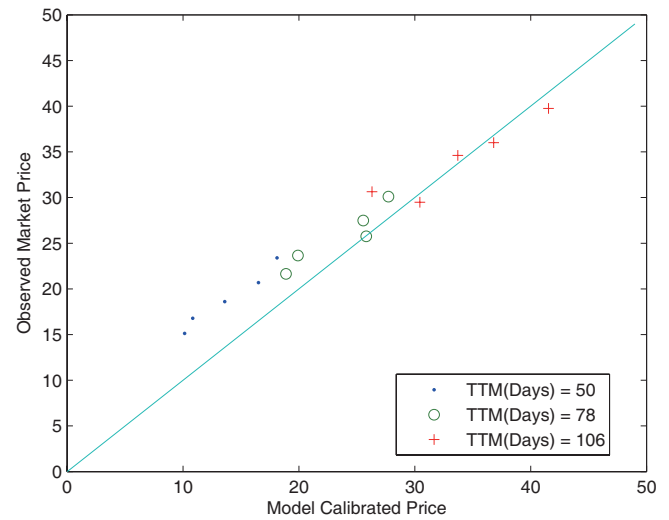

Figure 7 Model calibrated option prices and observed prices

Once the volatility is estimated from the underlying prices, we may start to calibrate the volatility premium from market option prices. We also implemented a gradient-based approach to minimize the normalized Mean Squared Error (MSE) of the model price and market observed price. Since the pricing algorithm is quite time-consuming. In our empirical study, we fit only one day option prices, namely 15 options from 5 different strikes and 3 time to maturities.

Figure 7 shows the calibrated result. We observe that the volatility premium $\lambda^{\mathbb{Q}}$ is $-5.882 \%$, which we think is reasonable because the more negative $\lambda^{\mathbb{Q}}$ is, the bigger the price would be since Vega is usually positive, which incorporate our assumptions that traders requires additional premium from the uncertainty from volatility. 


\section{Conclusion}

we employ particle filter method in American option pricing, with applications to real market data. SIR and APF algorithm and APFm are deeply explored. Numerical result shows that particle filter provides a good approximation to latent volatility and jumps for the SV, SVJ and SVCJ model. Empirical results suggest that the out of sample performance of particle filter based algorithm is stable consistency with observations. We also observe that a non-constant volatility risk premium is highly probable in the market as is introduced by the inconsistency between options on different maturities.

\section{References}

[1] Cheang G H, Chiarella C, Ziogas A. The representation of American options prices under stochastic volatility and jump-diffusion dynamics. Quantitative Finance, 2013, 13(2): 241-253.

[2] Chernov M, Ghysels E, Gallant A, et al. Alternative models for stock price dynamics. Journal of Econometrics, 2003, 116: 225-257.

[3] Eraker B, Johannes M, Polson N. The impact of jumps in volatility and returns. Journal of Finance, 2003, 59: 227-260.

[4] Harrison J M, Pliska S R. Martingales and stochastic integrals in the theory of continuous trading. Stochastic Processes and their Applications, 1981, 11: 215-260.

[5] Johannes M, Polson N, Stroud J. Optimal filtering of jump-diffusions: Extracting latent states from asset prices. Review of Financial Studies, 2007, 22(7): 2759-2799.

[6] Le A, Cen Z, Xu A. A robust upwind difference scheme for pricing perpetual American put options under stochastic volatility. International Journal of Computer Mathematics, 2012, 89(9): 1135-1144.

[7] Merton R C. Option pricing when the underlying stocks are discontinuous. Journal of Financial Economics, 1976, 5: 125-144.

[8] Pitt M K, Shephard N. Filtering via simulation: Auxiliary particle filters. Journal of the American Statistical Association, 1999, 94(446): 590-599.

[9] Rambharat B R, Brockwell A E. Sequential Monte Carlo pricing of American-style options under stochastic volatility models. The Annals of Applied Statistics, 2005, 4(1): 222-265.

[10] Saib A A E F, Tangman D Y, Bhuruth M. A new radial basis functions method for pricing American options under Merton's jump-diffusion model. International Journal of Computer Mathematics, 2012, 89(9): 11641185 .

[11] Salmi S, Toivanen J. Comparison and survey of finite difference methods for pricing American options under finite activity jump-diffusion models. International Journal of Computer Mathematics, 2012, 89(9): 1112-1134.

[12] Ye F, Zhou E. Pricing American options under partial observation of stochastic volatility. 2011 Winter Simulation Conference, 2011.

[13] Yun J. Density forecasting performances of the affine jump diffusion models using option-implied volatilities. Working Paper, 2012. 